\title{
Wavelength conversion in shortest-path all-optical networks
}

\section{Report}

Author(s):

Erlebach, Thomas; Stefanakos, Stamatis

Publication date:

2003-08

Permanent link:

https://doi.org/10.3929/ethz-a-004723738

Rights / license:

In Copyright - Non-Commercial Use Permitted

Originally published in:

TIK Report 177 
Thomas Erlebach, Stamatis Stefanakos

Wavelength Conversion in Shortest-Path

All-Optical Networks

TIK-Report

Nr. 177, August 2003 
Thomas Erlebach, Stamatis Stefanakos

Wavelength Conversion in Shortest-Path All-Optical Networks August 2003

TIK-Report Nr. 177

Computer Engineering and Networks Laboratory,

Swiss Federal Institute of Technology (ETH) Zurich

Institut für Technische Informatik und Kommunikationsnetze, Eidgenössische Technische Hochschule Zürich

Gloriastrasse 35, ETH Zentrum, CH-8092 Zürich, Switzerland 


\title{
Wavelength Conversion in Shortest-Path All-Optical Networks*
}

\author{
Thomas Erlebach Stamatis Stefanakos \\ Computer Engineering and Networks Laboratory (TIK) \\ ETH Zürich, CH-8092 Zürich, Switzerland \\ \{erlebach|stefanak\}@tik.ee.ethz.ch
}

\begin{abstract}
We consider all-optical networks with shortest-path routing that use wavelengthdivision multiplexing and employ wavelength conversion at specific nodes in order to maximize their capacity usage. We present efficient algorithms for deciding whether a placement of wavelength converters allows the network to run at maximum capacity, and for finding an optimal wavelength assignment when such a placement of converters is known. Our algorithms apply to both undirected and directed networks. Furthermore, we show that the problem of finding an optimal placement of converters is MAX SNP-hard in both undirected and directed networks. Finally, we give a linear-time algorithm for finding an optimal placement of converters in undirected triangle-free networks, and show that the problem remains $\mathcal{N} \mathcal{P}$-hard in bidirected triangle-free planar networks.
\end{abstract}

\section{Introduction}

All-optical networks are emerging as a promising solution for meeting the rapidly increasing bandwidth demand. In these networks optical switches are employed to avoid the bottleneck of opto-electronic conversions, and wavelength-division multiplexing is used to partition the optical bandwidth into channels that carry data at rates manageable by electronic network elements. A connection between two nodes must be carried through a single channel that operates on a different wavelength than any other channel with which it shares a fiber. Wavelength converters are being used to fully exploit the available capacity of the network. A converter, when placed at some node of the network, has the ability of altering the operating wavelength of any channel that goes through that node.

* Research partially supported by the Swiss National Science Foundation under Contract No. 2163563.00 (Project AAPCN) and the EU Thematic Network APPOL II (IST-2001-32007), with funding provided by the Swiss Federal Office for Education and Science (BBW). 
Several interesting algorithmic problems arise in such networks. The wavelength assignment problem asks for an assignment of a minimum number of wavelengths to a given set of connections such that no conflicts occur. If wavelength converters are used, the wavelength of a connection can change whenever its route goes through a converter, otherwise each connection must be carried through the same wavelength all the way from sender to receiver. The use of wavelength converters can decrease the number of necessary wavelengths for routing a given number of connections. Ideally, one should be able to route any set of connections that induce congestion $L$ (i.e., at most $L$ connections share a common fiber) with $L$ wavelengths. This is the case when all the nodes of the network are equipped with converters. However, due to the high cost of such devices one is faced with a network design problem, namely to achieve the optimal capacity usage by placing a minimum number of converters on the network.

In this paper, we study issues related to the design of networks with wavelength converters and to the problem of wavelength assignment in networks that already have conversion capabilities. We restrict ourselves to the practical scenario where shortest-path routing is used in the network; if one does not take into account the specific routing algorithm that will be used, and allows arbitrary routings, the requirements of converters are overly pessimistic.

Preliminaries. We model the network by a graph $G=(V, E)$. We will consider both undirected and directed graph models since both are of interest for optical networks. If the fiber allows two-way communication then we model the network as an undirected graph, while if the fiber allows one-way communication we model the network as a directed graph. A special case of directed graphs that are of particular interest are bidirected graphs: In these graphs, for every directed edge the oppositely directed edge also exists. Connections in the network can be seen as paths on $G$, and wavelengths can be regarded as colors. If the network does not employ wavelength conversion then a wavelength assignment or coloring for a set $\mathcal{P}$ of paths is an assignment of a color to each path in $\mathcal{P}$. A valid coloring is one in which no two paths that use the same edge get assigned the same color. In the presence of wavelength converters, a coloring is an assignment of a color to every edge of each path. If the converters are placed in the vertices in $S \subseteq V$ we say that a coloring is valid with respect to $S$ if it satisfies the additional constraint that the color assignments to two consecutive edges of a path differ only if their incident vertex is in $S$. We denote the load or congestion of the network that is induced by a set of paths $\mathcal{P}$ by $L(\mathcal{P})=\max _{e \in E} L_{e}(\mathcal{P})$ where $L_{e}(\mathcal{P})$ is the number of paths in $\mathcal{P}$ that use edge $e$. We denote the degree of a vertex $v$ by $\operatorname{deg}(v)$, and the maximum degree of $G$ by $\Delta(G)$ (in a directed graph, the degree of a vertex is its degree in the underlying undirected graph). The diameter of a graph is the number of edges on the longest shortest path. For any graph-theoretic terms or notation not defined here we refer the reader to [3].

Most networks that do not employ wavelength conversion are destined to waste capacity. Consider an undirected graph with a vertex $v$ of degree at least 3 , and assume that only 2 wavelengths are available. The paths between 3 neighbors of $v$ that go through $v$ have load 2 , but require 3 colors for a valid coloring if $v$ is not equipped with a converter. Therefore, 
not all 3 paths can be simultaneously routed, and furthermore, two of the edges incident to $v$ will only use one of the two available wavelengths resulting in a $50 \%$ waste of capacity. To avoid this, for a graph $G=(V, E)$ we need a placement of wavelength converters on a subset of its vertices $S \subseteq V$ such that any set of paths $\mathcal{P}$ on $G$ can be colored with $L(\mathcal{P})$ colors with respect to $S$. A set of vertices $S$ that has this property is a sufficient set. If the network uses shortest-path routing, we require that any set $\mathcal{P}$ of shortest paths can be colored with $L(\mathcal{P})$ colors with respect to $S$. We refer to this kind of sufficient sets for shortest-path routings as SP-sufficient sets. Note that an SP-sufficient set might be substantially smaller than a sufficient set. For example, a vertex of degree greater than or equal to 3 might not require a converter if its neighborhood does not contain an independent set of size three. We say that an induced $K_{1,3}$ is a claw; the vertex adjacent to the independent set of size three is its center.

Previous Work. Wilfong and Winkler [16] showed that the only bidirected graphs that admit the empty sufficient set are spiders, i.e., trees with at most one vertex of degree greater than two, and that rings (cycles) admit a sufficient set of size 1. They provided an efficient way of determining whether a set $S$ is sufficient for a bidirected graph $G$ : One modifies $G$ by "exploding" each node $s \in S$ into degree-of-s-many copies, each of which is made adjacent to one of the old neighbors of $s$. $S$ is sufficient for $G$ if and only if every component of the graph obtained after this modification is a spider. Concerning the problem of finding a minimum sufficient set (Min SUFFICIENT SET), they showed that it is $\mathcal{N} \mathcal{P}$-hard even for planar bidirected graphs. Kleinberg and Kumar [11] showed how the "exploding" technique from [16] can be extended for arbitrary directed graphs. They also gave a 2-approximation algorithm for arbitrary directed graphs and a polynomial time approximation scheme for directed planar graphs. Their approach can be extended to give a linear time algorithm for Min Sufficient SET in directed graphs of bounded treewidth [4]. Sufficient sets in undirected graphs were studied in [4]. Therein, it was shown that lines (paths) are the only undirected graphs that do not need converters, and an optimal polynomial-time algorithm for finding a minimum sufficient set was given. Networks with shortest-path routings were considered in [5]. A complete characterization of the undirected graphs which admit the empty SP-sufficient set, as well as efficient optimal coloring algorithms for this class of graphs were given.

The characterization in [5] implies that the restriction to shortest-path routings can reduce the converter requirements of a given network significantly. Furthermore, the known algorithms for MiN SufficIEnT SET from [4] and [11] indicate that by allowing arbitrary routings we end up with overly pessimistic placements of converters since they can result in picking half or even all vertices of degree greater than 2. Because of the high cost of wavelength converters, and since optical networks typically employ simple fixed-routing strategies, i.e., for every pair of nodes there is a prespecified path through which all traffic between these nodes will be carried (see [17]), it is important to consider the problem of converter placement under such practical scenarios and avoid the traditional worst-case analysis for arbitrary paths. In this paper, we mainly consider networks with shortest-path routing, but as we will see the algorithms we propose can be adapted to work for other 
fixed routings as well. From the theoretical point of view, it is interesting to observe how the restriction to shortest-path routings changes the nature of the problem thus requiring completely different methods for tackling it than the ones used for Min Sufficient SET in $[4,11,16]$.

Our Results. In Section 2, we give a polynomial-time algorithm for deciding whether for a given graph $G=(V, E)$, a subset $S \subseteq V$ is SP-sufficient, and a polynomial-time algorithm for optimally coloring any set of shortest paths on $G$ with respect to a valid SP-sufficient set. Both results make use of an auxiliary graph that depends only on the shortest paths of length 2 that exist on $G$ and not on $G$ itself, and hence apply to both directed and undirected graphs. This generalizes the result of [5], and also extends it to the directed case. We note that the proof given here, although for a much more general result, is substantially simpler and more elegant than the one given in [5]. In their most general form, our algorithms apply to networks with other routings as well (for example arbitrary routings). The only restriction is that the set of allowed paths on the network must be closed under taking subpaths of length 2 and 3. We are thus able to unify the results for identifying sufficient sets in undirected and directed graphs from $[4,11,16]$.

In Section 3, we turn to the problem of finding a minimum SP-sufficient set (MIN SP-Sufficient SET). We show that the problem is MAX SNP-hard for undirected and directed graphs by providing L-reductions from EdGE BIPARTIZATION and VerTEX BIPARTIZATION respectively, i.e., the problems of deleting a minimum set of edges or vertices in order to make a given graph bipartite. We also show that Min SP-Sufficient SET can be solved optimally in linear time in undirected triangle-free graphs, but remains $\mathcal{N} \mathcal{P}$-hard for bidirected triangle-free planar graphs.

We conclude in Section 4 with a discussion on possible directions for future research.

Other Related Work. Similar topics, to the ones we study here, were addressed in [14]. It is shown that for any all-optical network there exists an integer $\mu$ such that if we add $\mu$ parallel optical fibers to every link, the network does not need wavelength conversion. Wavelength converters of bounded degree have also been studied. A converter of bounded degree does not have full conversion capabilities (as in the case we study here), but it can transform wavelength $i$ to only a few other specified wavelengths. We refer the reader to [1] and the references therein.

\section{Identifying Valid Placements of Converters}

Before we present the main result of this section we need to introduce some notation. For a graph $G=(V, E)$ let $\mathcal{P}_{G}$ be the set of all shortest paths on $G$. For a set $\mathcal{P}$ of paths on $G$ and a set $S \subseteq V$, let $\mathcal{P}(S)$ be the set of paths obtained from $\mathcal{P}$ after cutting every path at the vertices of $S$ that it contains. Clearly, a coloring of a set $\mathcal{P}$ of paths with respect to $S \subseteq V$ is a coloring of $\mathcal{P}(S)$. For a set $\mathcal{P}$ of shortest paths on $G$ let $T(\mathcal{P})$ be the multigraph on $E$ with one edge for every path of length 2 in $\mathcal{P}$, and one edge for every shortest path of length 2 that is contained in a path of length greater than or equal to 3 in $\mathcal{P}$.

The following theorem characterizes valid SP-sufficient sets. 


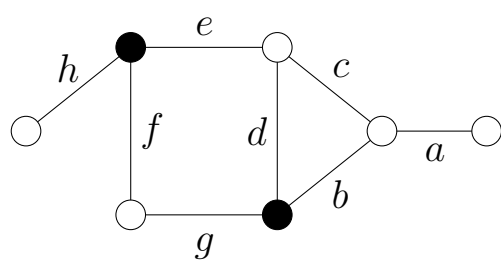

$G$

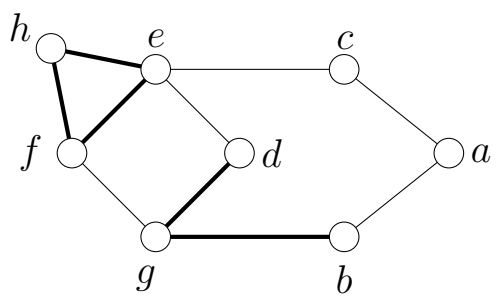

$T\left(\mathcal{P}_{G}(\emptyset)\right)$

Figure 1: An example of a graph $G$ along with $T\left(\mathcal{P}_{G}(S)\right)$ for $S=\emptyset$. Notice that both conditions of Theorem 1 are violated: $T\left(\mathcal{P}_{G}(\emptyset)\right)$ is not bipartite, and both edges of a shortest path of length 3 (e.g., the path using edges $a, c, e)$ lie consecutively on the same cycle. If we let $S$ consist of the two black vertices then $T\left(\mathcal{P}_{G}(S)\right)$ is obtained from $T\left(\mathcal{P}_{G}(\emptyset)\right)$ by removing the bold edges and $S$ is indeed an SP-sufficient set.

Theorem 1 Let $G=(V, E)$ be a (possibly directed) graph. $S \subseteq V$ is SP-sufficient for $G$ if and only if the following two conditions hold:

(i) $T\left(\mathcal{P}_{G}(S)\right)$ is bipartite, and

(ii) not both edges corresponding to a path of length 3 in $\mathcal{P}_{G}(S)$ lie consecutively on the same cycle in $T\left(\mathcal{P}_{G}(S)\right)$.

An example of the construction of $T\left(\mathcal{P}_{G}(S)\right)$ is shown in Fig. 1. An efficient algorithm for deciding whether a given subset $S \subseteq V$ is SP-sufficient for a graph $G=(V, E)$ follows easily. Constructing $T\left(\mathcal{P}_{G}(S)\right)$ and checking whether it is bipartite can clearly be done in polynomial-time. Verifying condition $(i i)$ can be done by checking for each path of length 3 whether its corresponding edges lie in different biconnected components of $T\left(\mathcal{P}_{G}(S)\right)$.

We proceed to prove Theorem 1 . It is easy to see that the conditions of the statement are indeed necessary. First assume that $T\left(\mathcal{P}_{G}(S)\right)$ is not bipartite. Then, $T\left(\mathcal{P}_{G}(S)\right)$ contains an odd cycle whose edges correspond to a set of shortest paths on $G$ with load 2 that require 3 colors for a valid coloring. To see that $(i i)$ is necessary, assume that $T\left(\mathcal{P}_{G}(S)\right)$ is bipartite and that there is a shortest path $p$ of length 3 in $\mathcal{P}_{G}(S)$ whose edges lie on the same (even) cycle $C$ in $T\left(\mathcal{P}_{G}(S)\right)$. Let $e$ be the middle edge of $p$. The paths corresponding to the edges of $C$ that are not incident to $e$, along with $p$, form a set of paths of load 2 that require 3 colors for a valid coloring. Sufficiency follows from the following theorem.

Theorem 2 Let $G=(V, E)$ be a (possibly directed) graph and $S \subseteq V$ be such that the conditions of Theorem 1 are satisfied. There exists a polynomial-time algorithm for coloring any set $\mathcal{P}$ of shortest paths on $G$ with $L(\mathcal{P})$ colors with respect to $S$.

Proof. Let $G=(V, E)$ be a (possibly directed) graph, and let $S \subseteq V$ be such that conditions $(i),(i i)$ hold. We show how to color a set $\mathcal{P}$ of shortest paths on $G$ with $L(\mathcal{P})$ colors with respect to $S$. For ease of notation we let $\mathcal{P}$ be equal to $\mathcal{P}(S)$. Also, we remove 
all paths of length one from $\mathcal{P}$ since these can be colored greedily afterwards. The coloring of $\mathcal{P}$ will be obtained by edge-coloring $T(\mathcal{P})$. The edge-coloring of $T(\mathcal{P})$ will be done by computing many local edge-colorings and then merging them in order to obtain a coloring for $T(\mathcal{P})$ with $L(\mathcal{P})$ colors such that edges in $T(\mathcal{P})$ that correspond to the same path in $\mathcal{P}$ get the same color.

We refer to paths in $\mathcal{P}$ of length 2 as short paths and to paths in $\mathcal{P}$ of length greater than or equal to 3 as long paths. We refer to edges in $T(\mathcal{P})$ that correspond to short paths as solid edges and to edges that correspond to paths of length 2 that are contained in some long path as partial edges. We call two partial edges that correspond to the same path in $\mathcal{P}$ relatives. An edge-coloring of $T(\mathcal{P})$ is valid if all edges incident to the same vertex get different colors, except for relative partial edges which are required to have the same color. Let $B_{1}, \ldots, B_{k}$ be the blocks (biconnected components) of $T(\mathcal{P})$ ordered according to a depth-first search traversal of the block graph of $T(\mathcal{P})$, i.e., the bipartite graph on $C \cup \mathcal{B}$ and edges $c B$ for $c \in C, B \in \mathcal{B}$ if $c \in B$, where $C$ is the set of cut-vertices of $T(\mathcal{P})$ and $\mathcal{B}$ is the set of blocks of $T(\mathcal{P})$. Let $H_{i}$ be the subgraph of $T(\mathcal{P})$ that contains all edges that are contained in block $B_{i}$ and all solid edges incident to a cut-vertex of $B_{i}$ that are not contained in $B_{i}$. Let $\mathcal{P}_{i} \subseteq \mathcal{P}$ be the set of paths that correspond to an edge in $H_{i}$. Since condition $(i)$ is satisfied, $H_{i}$ is bipartite and can be edge-colored with $\Delta\left(H_{i}\right)$ colors in polynomial time [12]. Since condition $(i i)$ is satisfied, $H_{i}$ may contain only one partial edge from each long path and hence the edge-coloring is valid for $H_{i}$. Furthermore, the degree of every vertex of $H_{i}$ is equal to the load of the corresponding edge in $G$ that is induced by $\mathcal{P}_{i}$.

Let $A_{i}$ be a valid edge-coloring for $H_{i}$ that uses $L\left(\mathcal{P}_{i}\right)$ colors. We will show how we can merge colorings $A_{1}, \ldots, A_{k}$ into a valid coloring for $\bigcup_{1 \leq i \leq k} H_{i}$ that uses $L(\mathcal{P})$ colors. We begin by merging $A_{1}$ with $A_{2}$. After the merging, $A_{2}$ is the new coloring we obtain. After having merged colorings $A_{1}, \ldots, A_{i-1}$ we proceed to the merging of $A_{i}$ with $A_{i-1}$. Define $H_{i}^{\prime}:=H_{1} \cup \cdots \cup H_{i}$. Let $\mathcal{P}_{i}^{\prime} \subseteq \mathcal{P}$ be the set of short paths that correspond to solid edges in $H_{i}^{\prime}$ and long paths that correspond to some partial edge in $H_{i}^{\prime}$. During the whole merging process we maintain the following invariants for $A_{i}$ : (a) $A_{i}$ uses no more than $L\left(\mathcal{P}_{i}^{\prime}\right)$ colors, and $(b) A_{i}$ is valid for $H_{i}^{\prime}$. After $k-1$ mergings, $H_{k}^{\prime}=T(\mathcal{P})$ and hence we will have obtained a valid coloring of $\mathcal{P}$ with $L(\mathcal{P})$ colors.

Assume we have merged the first $i-1$ colorings and the invariants $(a),(b)$ hold for $A_{i-1}$. We show how to merge $A_{i-1}$ with $A_{i}$ such that $(a),(b)$ are maintained. Let $x$ be the common cut-vertex of $B_{i}$ with a block whose coloring has already been merged. All solid edges incident to $x$ are colored with different colors in both $A_{i-1}$ and $A_{i}$. All partial edges incident to $x$ are colored in at most one of the two colorings. In order to merge the two colorings and maintain the invariants we have to ensure that (1) solid edges incident to $x$ have the same color in both $A_{i-1}$ and $A_{i}$, (2) partial edges incident to $x$ that correspond to the same path in $\mathcal{P}$ get the same color in $A_{i-1}$ and $A_{i}$, and (3) partial edges incident to $x$ that correspond to different paths in $\mathcal{P}$ get different colors in $A_{i-1}$ and $A_{i}$.

In order to ensure (1), we permute $A_{i}$ so that every solid edge incident to $x$ that is colored with different colors in $A_{i-1}$ and $A_{i}$ gets the color it has in $A_{i-1}$. In order to ensure (2), we permute $A_{i}$ so that every partial edge incident to $x$ that is colored in $A_{i}$ and is 


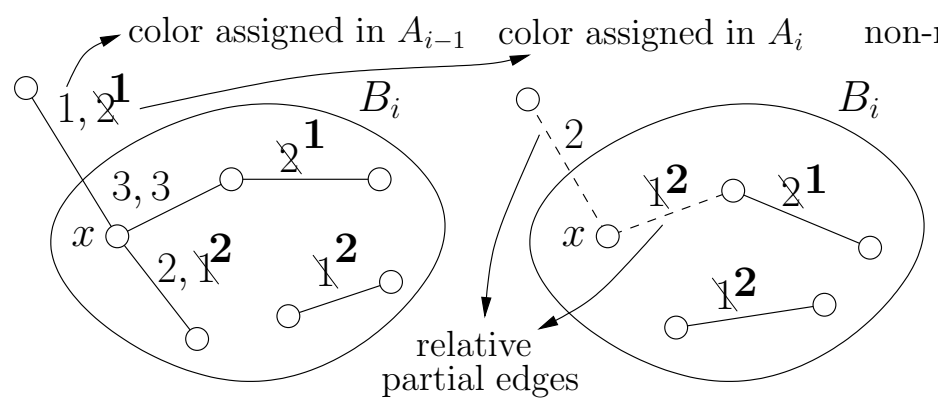

(1)

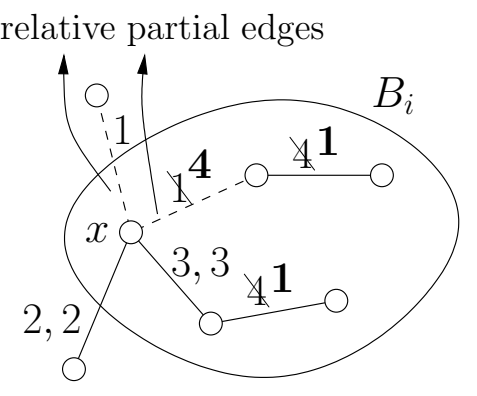

(3)

Figure 2: Example of the three types of permutations needed to maintain invariants (1),(2), and (3) in the proof of Theorem 2. Colors assigned in $A_{i}$ that change are crossed out and the new ones appear in bold.

relative to a partial edge $f$ colored in $A_{i-1}$, gets the color that $f$ has in $A_{i-1}$. We now show how we can ensure (3). Assume there are two partial edges $e$, colored in $A_{i-1}$, and $f$, colored in $A_{i}$, that have the same color and correspond to different paths. In $H_{i}^{\prime}$, these edges contribute 2 to the degree of $x$ (and their corresponding paths contribute 2 to the load of edge $x$ ). However, in each of $H_{i-1}^{\prime}, H_{i}$ only one of these edges is present. Therefore, there is at least one color among $1, \ldots, L\left(\mathcal{P}_{i}^{\prime}\right)$ that is not used by the edges incident to $x$ in $H_{i}^{\prime}$. Let $c$ be such a color. We assign color $c$ to $f$ and permute coloring $A_{i}$ so that all edges that were previously assigned color $c$ get the color that $f$ had and vice versa. Examples of the necessary modifications are shown in Fig. 2.

Since both colorings were valid, they remain valid after these modifications. Notice that since partial edges are only colored in one coloring it can happen that a partial and a solid edge have the same color in different colorings. However, since solid edges are colored in both colorings, after the permutation both edges will be colored with different colors. After permuting $A_{i}$, the two colorings become compatible and can be merged in the obvious way. After $k-1$ such mergings, $A_{k}$ is a valid coloring for $\mathcal{P}$ that uses $L(\mathcal{P})$ colors.

Notice that we have actually proved a stronger result. The fact that we are dealing with shortest paths does not play a role in the proof of Theorem 2. What we need is that $\mathcal{P}_{G}(S)$ in the conditions of Theorem 1 is closed under taking subpaths of length 2 and 3 : These are the paths needed for the necessity of the conditions in Theorem 1 since they provide the routings that witness the non-existence of a coloring with $L(\mathcal{P})$ colors. It is also interesting to observe that we can always find such a witness routing that has load 2 and requires 3 colors for a valid coloring, and that if no such routing exists then we can find a "good" coloring of any set of paths just by merging colorings of paths of length 2 .

Therefore, our algorithms for identifying whether a placement of converters allows a network to run at maximum capacity and for finding an optimal wavelength assignment with respect to such a placement of converters can be applied to any network that uses a routing with this property. If, for example, we allow arbitrary routings, we choose $\mathcal{P}_{G}$ as the set of all simple paths (actually the set of all simple paths of length 2 and 3 would 
do), thus providing an algorithm to unify the results in $[4,11,16]$ for identifying sufficient sets and finding an optimal wavelength assignment with respect to a valid sufficient set in both undirected and directed networks. Observe that in this case $T\left(\mathcal{P}_{G}\right)$ is simply the line graph of $G$ if we disregard parallel edges.

We also give an alternative formulation of Theorems 1 and 2 that might find applications in other problems as well.

Corollary 1 There exists a polynomial-time algorithm to decide whether for a given multiset $\mathcal{P}$ of paths, that is closed under taking subpaths of length 2 and 3 , all subsets $\mathcal{P}^{\prime} \subseteq \mathcal{P}$ can be colored with $L\left(\mathcal{P}^{\prime}\right)$ colors, and, if the answer is yes, a polynomial-time algorithm to find such a color assignment for any given $\mathcal{P}^{\prime} \subseteq \mathcal{P}$.

That is, given a set $\mathcal{P}$ of paths, with the property that for every path $p \in \mathcal{P}$ all its subpaths of length 2 and 3 are also in $\mathcal{P}$, we construct $T(\mathcal{P})$ and check whether the conditions of Theorem 1 are satisfied. If not then there exists some $\mathcal{P}^{\prime} \subseteq \mathcal{P}$ that can not be colored with $L\left(\mathcal{P}^{\prime}\right)$ colors. If the conditions are satisfied, any $\mathcal{P}^{\prime} \subseteq \mathcal{P}$ can be colored with $L\left(\mathcal{P}^{\prime}\right)$ colors using the algorithm described in the proof of Theorem 2.

\section{Complexity and Hardness of Placing Converters}

We now consider the problem of placing as few converters as possible on a given network in order to optimize its capacity usage. In order to show that this problem is hard even to approximate, we provide L-reductions (see [15]) from already known hard problems. For completeness we give here the definition of an L-reduction. An optimization problem $\Pi$ L-reduces to an optimization problem $\Pi^{\prime}$ if there are polynomial-time algorithms $f, g$, and constants $c_{1}, c_{2}$ such that for each instance $I$ of $\Pi(i)$ algorithm $f$ produces an instance $I^{\prime}=f(I)$ of $\Pi^{\prime}$, such that the cost of the optimal solutions of $I$ and $I^{\prime}, O P T(I)$ and $O P T\left(I^{\prime}\right)$, respectively, satisfy $O P T\left(I^{\prime}\right) \leq c_{1} O P T(I)$, and (ii) given any solution of $I^{\prime}$ with cost $S O L\left(I^{\prime}\right)$, algorithm $g$ produces a solution of $I$ with cost $S O L(I)$ such that $|S O L(I)-O P T(I)| \leq c_{2}\left|S O L\left(I^{\prime}\right)-O P T\left(I^{\prime}\right)\right|$.

\subsection{The Undirected Case}

Theorem 3 There exists an L-reduction from EDGE BIPARTIZATION to the undirected Min SP-Sufficient SET problem.

Proof. Let $G=(V, E)$ be an instance of Edge Bipartization, and assume, without loss of generality, that $G$ does not contain any vertices of degree 1 . We first show how to construct an undirected instance $G^{\prime}=\left(V^{\prime}, E^{\prime}\right)$ of Min SP-Sufficient Set. The idea behind the construction is to map odd cycles of $G$ onto "bad" induced odd cycles in $G^{\prime}$, i.e., cycles on which the set of all shortest paths of length 2 corresponds to an odd cycle in $T\left(\mathcal{P}_{G}(\emptyset)\right)$. Since single triangles do not allow the construction of such a set of shortest paths, but have to be hit by any solution of EDGE BIPARTIZATION, we will replace each 

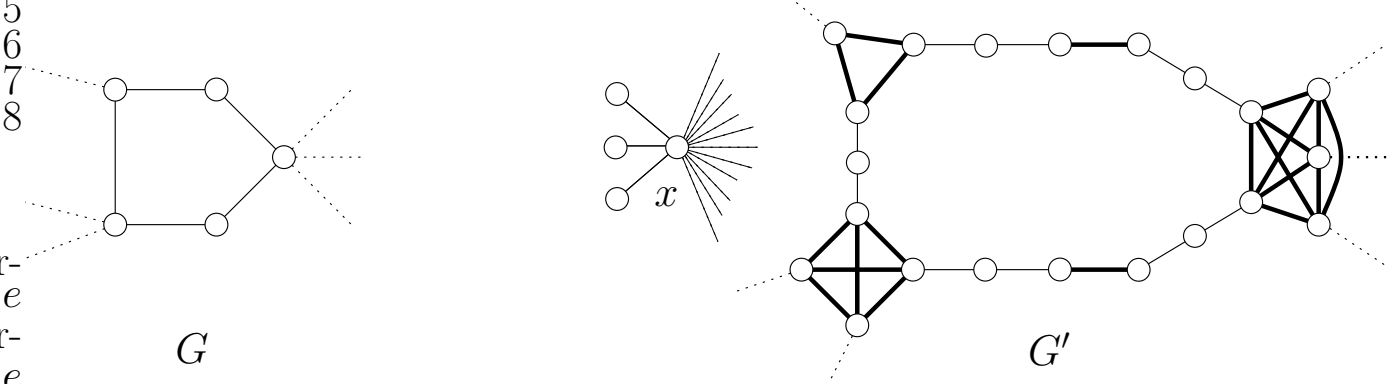

Figure 3: The transformation used in the reduction in the proof of Theorem 3. Bold edges are in cliques that correspond to vertices of $G$.

edge with a long path in order to "blow up" the length of the cycles of $G$. Moreover, in order to avoid claws in $G^{\prime}$ we will use a special gadget for every vertex of $G$. Finally, we will eliminate all shortest paths of length 3 from $G^{\prime}$ so that no other configuration apart from odd induced cycles of length greater than 3 will need to be hit by a solution for MiN SP-Sufficient Set.

We construct $G^{\prime}$ as follows. For each vertex $v$ of $\operatorname{degree} \operatorname{deg}(v)$ in $G$ we have a clique on $\operatorname{deg}(v)$ vertices. For each edge $u v$ in $G$ we have a simple path on 3 vertices. The endpoints of the path are identified with two distinct vertices in the cliques that correspond to $u$ and $v$. The construction of $G^{\prime}$ is completed by adding a claw with center $x$ and making $x$ adjacent to all other vertices. Observe that the parity of each cycle in $G$ is preserved in $G^{\prime}$ : a cycle on $m$ edges in $G$ is mapped onto an induced cycle on $3 m$ edges in $G^{\prime}$. An example is shown in Fig. 3.

Consider an optimal solution to the instance of EDGE BipARTizATion, namely a set of edges $F \subseteq E$ of minimum cardinality $O P T_{\mathrm{BIP}}$ such that $G-F$ is bipartite. We construct an SP-sufficient set $S$ of size $O P T_{\mathrm{BIP}}+1$ for $G^{\prime}$ as follows. For every edge $e \in F$ we take in $S$ one of the endpoints of its corresponding path in $G^{\prime}$. Furthermore, we take $x$ into $S$ since it is the center of a claw. We will show that $S$ is indeed an SP-sufficient set. Since $G^{\prime}$ has diameter 2 , according to Theorem 1 we only need to show that $T\left(\mathcal{P}_{G^{\prime}}(S)\right)$ is bipartite, or equivalently that the edges of $G^{\prime}$ can be 2-colored such that no shortest path of length 2 that does not go through a converter uses edges of the same color. We exhibit such a coloring. Consider a bipartition of $G-F$. For every vertex that is in the one side of the partition we color the edges of its corresponding clique in $G^{\prime}$ with color $\alpha$. All edges in cliques corresponding to vertices in the other side of the partition are colored with color $\beta$. We extend this coloring to paths corresponding to edges in $E \backslash F$. Such paths connect cliques whose edges get different colors. Therefore, we can assign to each of the two edges of such a path a different color than the one that its incident clique already has. Paths corresponding to edges in $F$ connect cliques assigned the same color. Consider a path connecting two cliques with the same color, say $\alpha$. Since one of the endpoints of the path is in $S$, we assign color $\alpha$ to the edge of the path incident to the converter and color $\beta$ to 
the other edge. Finally, we assign the same color, say $\alpha$, to all edges incident to $x$. It is easy to see that the only shortest paths of length 2 that use edges of the same color are the ones that go over converters. Therefore, $S$ is an SP-sufficient set.

Now, assume we are given an SP-sufficient set $S^{\prime}$ and consider a 2-coloring of the edges of $G^{\prime}$, with colors $\alpha, \beta$, such that no shortest path of length 2 that does not go over a converter uses edges of the same color. We can assume that all edges of some clique corresponding to a vertex in $G$ have the same color. If this is not the case we can transform the coloring into one that has this property as follows. Consider a clique $K$ in $G^{\prime}$, corresponding to some vertex in $G$, whose edges are not monochromatic. If all the vertices of the clique are in $S^{\prime}$ then we can simply modify the coloring of the edges of $K$ so that they all have the same color. The resulting coloring still has the property that no shortest path of length 2 that does not go over a converter uses edges of the same color. Otherwise, there is some vertex $v \in K$ without a converter. Say that its incident edge outside $K$ has color $\alpha$. Then, all its incident edges in $K$ must have color $\beta$. Consider now some edge $u w$ in $K$ that has color $\alpha$. Let $u^{\prime}, w^{\prime}$ be the neighbors of $u$ and $w$, respectively, outside $K$. The paths $v u u^{\prime}, u^{\prime} u w$ are shortest and whatever the color assigned to edge $u u^{\prime}$, one of the two paths will use two edges of the same color. Therefore, $u$ is in $S^{\prime}$. Similarly, because of the paths $v w w^{\prime}, w^{\prime} w u$, $w$ is also in $S^{\prime}$. Hence, if we change the color of $u w$ to $\beta$ the coloring still has the desired property. By repeating this modification we can obtain a coloring such that all cliques are monochromatic, and thus our assumption is without loss of generality.

Construct a solution $F^{\prime}$ to Edge BipartizAtion by taking all the edges whose corresponding path in $G^{\prime}$ contains a vertex in $S^{\prime}$. We show that $G-F^{\prime}$ is indeed bipartite. Construct a bipartition by taking in one side vertices corresponding to cliques in $G^{\prime}$ whose edges are colored with color $\alpha$. The other side contains the remaining vertices. Assume for a contradiction that there is an edge $e \in E \backslash F^{\prime}$ whose endpoints are in the same side. Then there can not exist a valid 2-coloring of the edges of the path corresponding to $e$, a contradiction. Note that $\left|F^{\prime}\right| \leq\left|S^{\prime}\right|-1$.

Let $O P T_{\mathrm{SPSS}}$ denote the size of an optimal SP-sufficient set for $G^{\prime}$. Since from a given SP-sufficient set $S^{\prime}$ we can obtain a solution to EDGE BipartizAtion $F^{\prime}$ of size at most $\left|S^{\prime}\right|-1$, and from an optimal solution to EDGE Bipartization we can obtain an SPsufficient set of size $O P T_{\mathrm{BIP}}+1$, it follows that $O P T_{\mathrm{SPSS}}=O P T_{\mathrm{BIP}}+1$. Furthermore, $\left|F^{\prime}\right|-O P T_{\mathrm{BIP}} \leq\left|S^{\prime}\right|-O P T_{\mathrm{SPSS}}$ and therefore the transformation is an L-reduction.

Since Edge Bipartization is known to be MAX SNP-hard [15], and since the constructed instance of Min SP-Sufficient SET has diameter 2, we obtain the following corollary.

Corollary 2 The undirected Min SP-SufficiEnt SET problem is MAX SNP-hard even when restricted to graphs of diameter 2.

Observe that the presence of triangles in the proof of Theorem 3 is essential in order to avoid claws. In fact, if we restrict to triangle-free graphs then the problem can be solved optimally in polynomial time as the following theorem illustrates.

Theorem 4 There exists a linear-time algorithm for finding a minimum SP-sufficient set in undirected triangle-free graphs. 
Proof. Consider an undirected triangle-free graph $G=(V, E)$, and assume, without loss of generality, that $G$ is connected and is not a cycle (in the latter case, a single converter at an arbitrary node of the cycle is SP-sufficient except for a cycle on 4 vertices where the empty set suffices, see [16]). Denote by $N(v)$ the subgraph induced by the vertices adjacent to $v$. Since $G$ is triangle-free, $N(v)$ is an independent set for all $v \in V$. Furthermore, any SP-sufficient set must contain all vertices $v \in V$ with $\operatorname{deg}(v) \geq 3$ since otherwise there would be a claw without a converter. The set $S=\{v \in V \mid \operatorname{deg}(v) \geq 3\}$ is a minimum SP-sufficient set: $V \backslash S$ contains vertices of degree at most 2, hence if we explode all vertices in $S$, as described in Section 1, the exploded graph is a collection of lines. By [4], $S$ is a sufficient set, therefore also SP-sufficient and the statement is proved.

\subsection{The Directed Case}

The directed Min SP-Sufficient SET problem gives us more freedom in designing a reduction since claws do not necessarily require a converter. This is in accord to our expecting the directed case to be more difficult (recall that Min Sufficient SET is $\mathcal{N} \mathcal{P}$ hard in directed graphs but polynomial in undirected). Indeed, in this case we are able to give an approximation preserving reduction from VERTEX BIPARTIZATION, which is likely to be harder than its edge-deletion counterpart (see [13]).

Theorem 5 There exists an L-reduction from VERTEX BIPARTIZATION to the directed Min SP-Sufficient SET problem.

Proof. The idea behind the reduction is the same as in the undirected case. The main difference, which simplifies the construction and allows us to reduce a vertex-deletion problem to Min SP-Sufficient Set, is that claws do not necessarily require a converter in the directed case; hence we do not need to use a special gadget for every vertex.

Let $G=(V, E)$ be an instance of Vertex Bipartization. The directed instance $G^{\prime}=\left(V^{\prime}, E^{\prime}\right)$ of Min SP-Sufficient SET is constructed as follows. For every vertex $v$ in $G$ we have a vertex $v^{\prime}$ in $G^{\prime} ; v^{\prime}$ will correspond to $v$. For every edge $u v$ in $G$ we have a bidirected path of length 3 between $u^{\prime}$ and $v^{\prime}$; each of the two directed paths (i.e., from $u^{\prime}$ to $v^{\prime}$ and from $v^{\prime}$ to $u^{\prime}$ ) will be called a blow-up path and will correspond to edge $u v$. Finally, we add a new vertex $x$ which is made adjacent to all other vertices via two oppositely directed edges.

Consider an optimal solution to the instance of Vertex BipARTizAtion, namely a set of vertices $U \subseteq V$ of minimum cardinality $O P T_{\mathrm{BIP}}$ such that $G-U$ is bipartite. We construct an SP-sufficient set $S$ of size $|U|$ for $G^{\prime}$ by taking all vertices corresponding to vertices in $U$. We show that $S$ is indeed an SP-sufficient set by exhibiting a 2-coloring of the edges of $G^{\prime}$, as in the proof of Theorem 3. Consider a bipartition of $G-U$ into a left and a right part. Vertices in $G^{\prime}$ that correspond to vertices in the left side of the partition are called left vertices. Vertices in $G^{\prime}$ that correspond to vertices in the right side of the partition are called right vertices. Vertices in $G^{\prime}$ that correspond to vertices in $U$ are called deleted vertices. Assign color $\alpha$ to edges directed out of left vertices and color 
$\beta$ to edges directed out of right vertices. Now, simply extend this coloring by assigning alternating colors to the edges of all blow-up paths that have one edge already colored. The only edges remaining uncolored are edges incident to $x$ and edges in blow-up paths directed out of deleted vertices. We color all edges directed into $x$ with $\alpha$ and all edges directed out of $x$ with $\beta$. All edges directed out of deleted vertices which are in blow-up paths that end up at a left vertex are colored with color $\beta$. The remaining edges directed out of deleted vertices are colored with color $\alpha$. The remaining blow-up paths that have uncolored edges (these are paths directed out of some deleted vertex) can be colored by assigning alternating colors to the uncolored edges depending on the color assigned to the edge incident to the deleted vertex. It is easy to see that the only shortest paths of length 2 that use edges of the same color are paths that go over vertices which correspond to vertices in $U$. Since these vertices are equipped with converters it follows that $S$ is an SP-sufficient set.

Now, assume we are given an SP-sufficient set $S^{\prime}$ for $G^{\prime}$. We can assume that $S^{\prime}$ consists only of vertices of degree greater than 2. Construct a solution $U^{\prime}$ to Vertex BipartizATION by taking all vertices that correspond to vertices in $S^{\prime}$. To see that $G-U^{\prime}$ is bipartite assume for a contradiction that it contains an odd cycle. Consider the blow-up paths in $G^{\prime}$ that correspond to the edges of the cycle: They form two oppositely directed odd cycles in $G^{\prime}$ which can be used for a construction of a set of paths with load 2 that requires 3 colors for a valid coloring. Hence, at least one of the vertices of the cycle is in $S$, and therefore its corresponding vertex in $G$ is deleted, a contradiction.

Let $O P T_{\text {SPSS }}$ denote the value of an optimal SP-sufficient set for $G^{\prime}$. Since from a given SP-sufficient set for $G^{\prime}$ we can construct a valid solution to VERTEX BipARTIZATION of the same size it follows that $O P T_{\mathrm{SPSS}} \geq O P T_{\mathrm{BIP}}$ and hence $|S|=O P T_{\mathrm{SPSS}}=O P T_{\mathrm{BIP}}$. Furthermore, $\left|U^{\prime}\right|-O P T_{\mathrm{BIP}} \leq\left|S^{\prime}\right|-O P T_{\mathrm{SPSS}}$ and hence the transformation is an Lreduction.

Since Vertex Bipartization is known to be MAX SNP-hard [13], and our constructed instance of Min SP-Sufficient SET is bidirected and has diameter 2, Theorem 5 implies the following.

Corollary 3 The directed Min SP-Sufficient SET problem is MAX SNP-hard even when restricted to bidirected graphs of diameter 2.

The following theorem shows that Min SP-Sufficient SET is $\mathcal{N} \mathcal{P}$-hard even on bidirected triangle-free planar graphs. The reduction is from PLANAR 3-SAT (see [7]) and is similar to the one given in [16] for showing the hardness of Min Sufficient SET. Our reduction shows that, in contrast to the undirected case, Min SP-Sufficient SET remains $\mathcal{N} \mathcal{P}$-hard even on triangle-free bidirected graphs.

Theorem 6 The problem of determining whether for a given directed graph $G$ and an integer $k$ there exists an SP-sufficient set for $G$ of size $k$ is $\mathcal{N} \mathcal{P}$-complete even when restricted to bidirected triangle-free planar graphs. 
Proof. We refer to the decision version of Min SP-Sufficient Set as SP-Sufficient SET. For a directed graph $G=(V, E)$ it is easy to check in polynomial time if $S \subseteq V$ is SP-sufficient as is shown in Theorem 1. Therefore, SP-Sufficient SET is in $\mathcal{N} \mathcal{P}$. The $\mathcal{N} \mathcal{P}$-hardness of SP-Sufficient SET follows by a reduction from the Planar 3SAT problem [7]. An instance of Planar 3SAT is a set of clauses $\left\{C_{1}, \ldots, C_{m}\right\}$, each with exactly 3 literals, with the additional property that the bipartite graph with a vertex for each variable and a vertex for each clause and with an edge between a variable vertex $x$ and clause vertex $C$ when $x$ or $\bar{x}$ is in the clause $C$ is planar. Given an instance $I$ of PlanAR 3SAT, we show how to construct an instance $I^{\prime}$ of SP-Sufficient SET that is a YES instance if and only if $I$ is satisfiable. The construction of $I^{\prime}$ will be polynomial in the size of $I$. The construction is the same as the one used for Sufficient SET in bidirected graphs [16]; the only difference is that we use a cycle of length 5 instead of a triangle for each clause because of the restriction to shortest paths.

Let $I$ be an instance of PLANAR 3SAT with $m$ clauses on $n$ variables, each clause containing exactly 3 literals. Construct a bidirected graph $G$ as follows. For ease of presentation the construction will be given for the skeleton of $G$, i.e., when we say "edge" (or "adjacent") we actually mean "(connected with) two oppositely directed edges." For each variable $x_{i}, G$ contains 2 vertices $x_{i}, \bar{x}_{i}$ adjacent to each other. We refer to these vertices as variable vertices. Two additional vertices are made adjacent to each variable vertex. For each clause a cycle of length 5 is added to $G$. For clause $C_{j}$, let $u_{j}^{1}, u_{j}^{2}, u_{j}^{3}$ be three consecutive vertices of the cycle corresponding to $C_{j}$. These three vertices will correspond to the literal occurrences in $C_{j}$ and will be called literal vertices. If $x_{i}$ occurs in $C_{j}$ then an edge is added between $x_{i}$ and the vertex corresponding to this literal occurrence; if $\bar{x}_{i}$ occurs then an edge is added between $\bar{x}_{i}$ and the corresponding vertex. These edges will be called the connecting edges. An example of the construction for $C_{1}=x_{1} \vee \bar{x}_{2} \vee \bar{x}_{3}$ is shown in Fig. 4. Clearly, $G$ is planar since $I$ is an instance of PLANAR 3SAT, and does not contain any triangles.

We claim that there is an SP-sufficient set $S$ for $G$ of $2 m+n$ vertices if and only if $I$ is satisfiable.

Suppose that $I$ is satisfiable and let $T$ be a satisfying truth assignment. We call a variable vertex true (resp. false) if its corresponding variable evaluates to true (resp. false) according to $T$. Let $S$ contain all true variable vertices and two out of the three literal vertices of each clause such that the literal vertex that is not taken in $S$ corresponds to a true literal occurrence (such a vertex exists since $T$ is satisfying). Notice that $|S|=2 m+n$. It is easy to see that after exploding all vertices in $S$, as described in Section 1, the resulting graph consists of spiders. Thus, by the result of Wilfong and Winkler [16], it follows that $S$ is a sufficient set and thus also an SP-sufficient set.

For the other direction we first need to show that any SP-sufficient set needs to contain at least one vertex from the gadget that was used to represent variables. Let $x, y$ be two adjacent vertices and let $a, b$ be adjacent to $x$ and $c, d$ be adjacent to $y$. Consider the set of directed paths $\mathcal{P}=\{a x b, a x y c, d y c, d y x, y x b\}$. These paths have load 2 but require 3 colors for a valid coloring. Hence, if all paths in $\mathcal{P}$ are shortest any SP-sufficient set will contain at least one of $x$ or $y$. Therefore, any SP-suffient set must contain $x_{i}$ or $\bar{x}_{i}$ for 


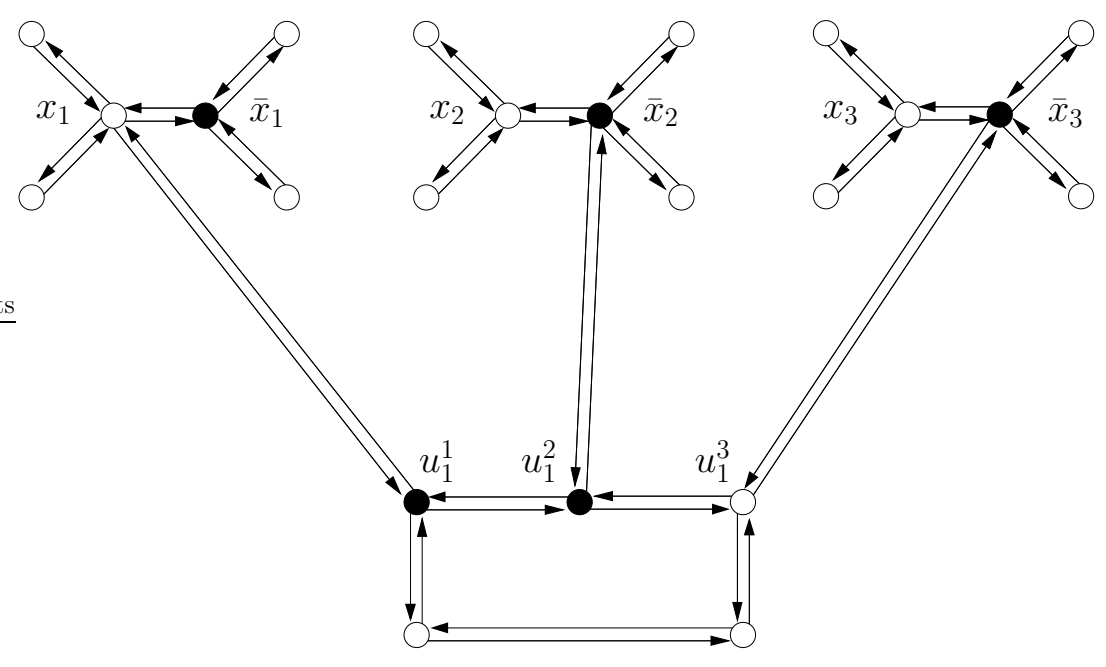

Figure 4: The construction used in the hardness proof for the directed case.

every variable $x_{i}$. In fact this construction can be generalized by connecting $x$ and $y$ with a bidirected path; in this case any SP-sufficient set must contain a vertex of the bidirected path from $x$ to $y$. We call this generalized configuration an $\mathcal{H}-$ path on $x$ and $y$.

Now, assume that $S$ is an SP-sufficient set of size $2 m+n$. $S$ must contain at least one variable vertex from each variable gadget. Also, because of the connecting edges, $S$ should contain at least 2 of the literal vertices of each clause gadget; otherwise there is an $\mathcal{H}$-path without a converter. This is easy to see if only $u_{j}^{1}$ or $u_{j}^{3}$ is in $S$. If only $u_{j}^{2}$ is in $S$ notice that there is a generalized $\mathcal{H}$-path on $u_{j}^{1}$ and $u_{j}^{3}$ (with $u_{j}^{2}$ adjacent to both $u_{j}^{1}$ and $u_{j}^{3}$ ) with 9 shortest paths on it with load 2 that require 3 colors for a valid coloring. Furthermore, we can assume, w.l.o.g., that $S$ contains $u_{j}^{1}$ or $u_{j}^{3}$; otherwise we move the converter from the vertex of the bidirected path between $u_{j}^{1}$ and $u_{j}^{3}$ to one of its endpoints and the $\mathcal{H}$-path is still hit. Therefore, since $|S|=2 m+n, S$ contains exactly one variable vertex from each variable gadget and exactly two literal vertices from each clause gadget. Define a truth assignment for $I$ by setting $x_{i}=1$ if $x_{i} \in S$ and $x_{i}=0$ otherwise. Consider a clause $C_{j}$. There is exactly one literal vertex in $G$ corresponding to a literal occurrence in $C_{j}$ that is not in $S$. However, the variable of this occurrence must be in $S$ since otherwise there is an $\mathcal{H}$-path without a converter. Therefore, there is at least one true literal in each clause and $T$ is a satisfying assignment.

\section{Discussion}

We presented a polynomial time algorithm for deciding whether for a given graph $G=$ $(V, E)$ a subset $S \subseteq V$ is SP-sufficient, and a polynomial-time algorithm for optimally coloring any set of shortest paths on $G$ with respect to an SP-sufficient set. These algorithms can be extended to address sufficient sets for arbitrary routings in both undirected 
and directed graphs. We also showed that Min SP-Sufficient SET is at least as hard to approximate as EDGE BIPARTIZATION in undirected graphs, and at least as hard to approximate as VERTEX BIPARTIZATION in directed graphs (and hence is MAX SNP-hard in both cases). Finally, we gave a linear-time algorithm that solves MiN SP-SufficIENT SET optimally in undirected triangle-free graphs and showed that the problem remains $\mathcal{N} \mathcal{P}$-hard even for bidirected triangle-free planar graphs.

A challenging remaining open problem is that of designing good approximation algorithms for MIN SP-SUFficIENT SET. Although we are able to prove only MAX SNPhardness of Min SP-Sufficient SET, our results indicate that it is unlikely or at least highly non-trivial to obtain a constant factor approximation algorithm. Indeed, that would imply a constant approximation for EdGE or VERTEX BIPARTIZATION whose approximability status has had a gap of $O(\log n)$ for the last 10 years $(\operatorname{an} O(\log n)$ approximation for both problems follows from the multicut algorithm of Garg, Vazirani and Yannakakis [8, 9], see also an older polylogarithmic approximation given in [10]). In [13], Lund and Yannakakis conjecture that for every nontrivial, hereditary property $\pi$ with an infinite number of minimal forbidden subgraphs the node-deletion problem cannot be approximated with constant ratio. Although the conjecture has been disproved for $\pi=$ "acyclic" (by a constant approximation for the undirected feedback vertex set problem [2]) and for properties derived from matroids definable on the edge set of any graph [6], it still remains likely that this is the case for VERTEX BIPARTIZATION.

Therefore, the design of a logarithmic approximation or of a reduction that places MiN SP-SufFICIENT SET in some higher inapproximability class appears to be the most promising direction for future research. From the algorithmic point of view, Min SP-SufficiEnT Set seems similar to Edge Bipartization since by placing converters in $G$ we delete certain edges in $T\left(\mathcal{P}_{G}(\emptyset)\right)$. We believe however that Min SP-Sufficient SET is actually harder than Edge BipartizAtion: The reason is that a single converter on some vertex $v$ does not delete one edge from $T\left(\mathcal{P}_{G}(\emptyset)\right)$ but all the edges that correspond to the shortest paths of length 2 that go through $v$ in $G$. If the edge-sets in $T\left(\mathcal{P}_{G}(\emptyset)\right)$ that correspond to single vertices in $G$ are connected subgraphs then a logarithmic approximation, at least for bipartizing $T\left(\mathcal{P}_{G}(\emptyset)\right)$, can be obtained by a simple adaptation of the multicut-based algorithm given in [9]. Unfortunately, this is not the case in general, in both undirected and directed graphs. Furthermore, solving Min SP-Sufficient SET requires not only bipartizing $T\left(\mathcal{P}_{G}(\emptyset)\right.$ ), but also hitting all "bad" paths of length 3 . In all our reductions we explicitly forced the diameter of the constructed graph to be less than 3 in order to focus on the bipartization phase of the problem and ignored the second condition of Theorem 1. We believe that these additional degrees of complexity that Min SP-Sufficient SET has, could be exploited in order to place it in some higher inapproximability class. We note finally that an $n / k$-approximation for any constant $k$ follows trivially from Theorem 1 : We search for a valid SP-sufficient set in all subsets up to $k$ vertices; if none is found we place a converter at every vertex. 


\section{References}

[1] V. Auletta, I. Caragiannis, L. Gargano, C. Kaklamanis, and P. Persiano. Sparse and limited wavelength conversion in all-optical tree networks. Theoretical Computer Science, 266(1-2):887-934, 2001.

[2] V. Bafna, P. Berman, and T. Fujito. A 2-approximation algorithm for the undirected feedback vertex set problem. SIAM Journal on Discrete Mathematics, 12(3):289-297, 1999.

[3] R. Diestel. Graph Theory. Springer-Verlag, 2000.

[4] T. Erlebach and S. Stefanakos. Wavelength conversion in networks of bounded treewidth. TIK-Report 132, ETH Zurich, April 2002.

[5] T. Erlebach and S. Stefanakos. On shortest-path all-optical networks without wavelength conversion requirements. In Proceedings of the 20th International Symposium on Theoretical Aspects of Computer Science (STACS'03), LNCS 2607, pages 133-144, 2003.

[6] T. Fujito. A primal-dual approach to approximation of node-deletion problems for matroidal properties. In Proccedings of the 24th International Colloquium on Automata, Languages, and Programming (ICALP'97), LNCS 1256, pages 749-759, 1997.

[7] M. R. Garey and D. S. Johnson. Computers and Intractability: A Guide to the Theory of NP-completeness. Freeman, 1979.

[8] N. Garg, V. V. Vazirani, and M. Yannakakis. Multiway cuts in directed and node weighted graphs. In Proccedings of the 21st International Colloquium on Automata, Languages, and Programming (ICALP'94), LNCS 820, pages 487-498, 1994.

[9] N. Garg, V. V. Vazirani, and M. Yannakakis. Approximate max-flow min-(multi)cut theorems and their applications. SIAM J. Comput., 25(2):235-251, 1996.

[10] P. N. Klein, S. Rao, A. Agrawal, and R. Ravi. An approximate max-flow mincut relation for unidirected multicommodity flow, with applications. Combinatorica, 15(2):187-202, 1995.

[11] J. Kleinberg and A. Kumar. Wavelength conversion in optical networks. Journal of Algorithms, 38(1):25-50, 2001.

[12] D. König. Über Graphen und ihre Anwendung auf Determinantentheorie und Mengenlehre. Math. Ann., 77:453-465, 1916.

[13] C. Lund and M. Yannakakis. The approximation of maximum subgraph problems. In Proccedings of the 20th International Colloquium on Automata, Languages, and Programming (ICALP'93), LNCS 700, pages 40-51, 1993. 
[14] L. Margara and J. Simon. Decidable properties of graphs of all-optical networks. In Proceedings of the 28th International Colloquium on Automata, Languages, and Programming (ICALP'01), LNCS 2076, pages 518-529, 2001.

[15] C. Papadimitriou and M. Yannakakis. Optimization, approximation, and complexity classes. Journal of Computer and System Sciences, 43:425-440, 1991.

[16] G. Wilfong and P. Winkler. Ring routing and wavelength translation. In Proceedings of the 9th Annual ACM-SIAM Symposium on Discrete Algorithms (SODA'98), pages 333-341, 1998.

[17] H. Zang, J. Jue, and B. Mukherjee. A review of routing and wavelength assignment approaches for wavelength-routed optical WDM networks. Optical Networks Magazine, 1(1):47-60, January 2000. 